\title{
Mantle cell lymphoma masked by posttraumatic
}

\section{pancreatic hematoma}
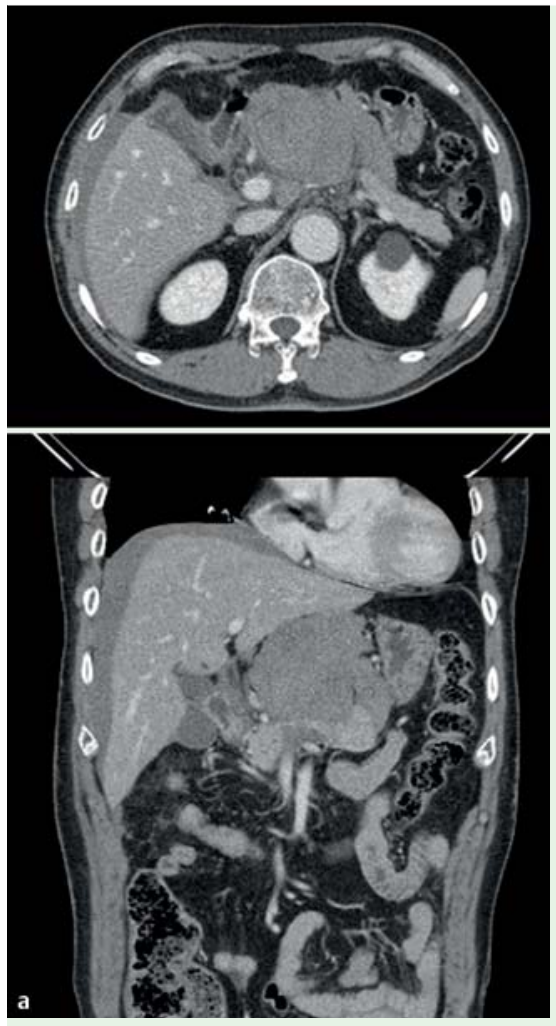

Fig. 1 Evolving peripancreatic lesion. a Abdominal computed tomography immediately after a car accident shows an enhancing peripancreatic mass. b After

6 months, the lesion has shrunk. c A huge peripancreatic mass is incidentally identified following appendectomy 18 months after the injury.

Pelvic computed tomography revealed a $15-\mathrm{cm}$ peripancreatic mass in a 73-yearold man undergoing an operation for appendicitis associated with a periappendiceal abscess. The patient had been hospitalized 18 months previously for blunt abdominal trauma sustained during a motor vehicle collision. At that time, hemoperitoneum and a peripancreatic hematoma were diagnosed ( Fig.1a). The hematoma decreased in size within 1 week, and there was no evidence of major pancreatic duct injury; thus, he was managed conservatively. The lesion continued to shrink for 6 months after the injury ( $\bullet$ Fig. 1 b), but the volume had increased at the time of the appendectomy, as shown by computed tomography ( $\bullet$ Fig. $1 \mathrm{c}$ ). Endoscopic ultrasound-guided biopsy with a 19-gauge needle (ProCore; Cook Medical, Bloomington, Indiana, USA) was used to identify the lesion. Pathologic examination and immunohistochemical staining of the specimen yielded a diagnosis of mantle cell lymphoma ( $\bullet$ Fig. 2 a-c).
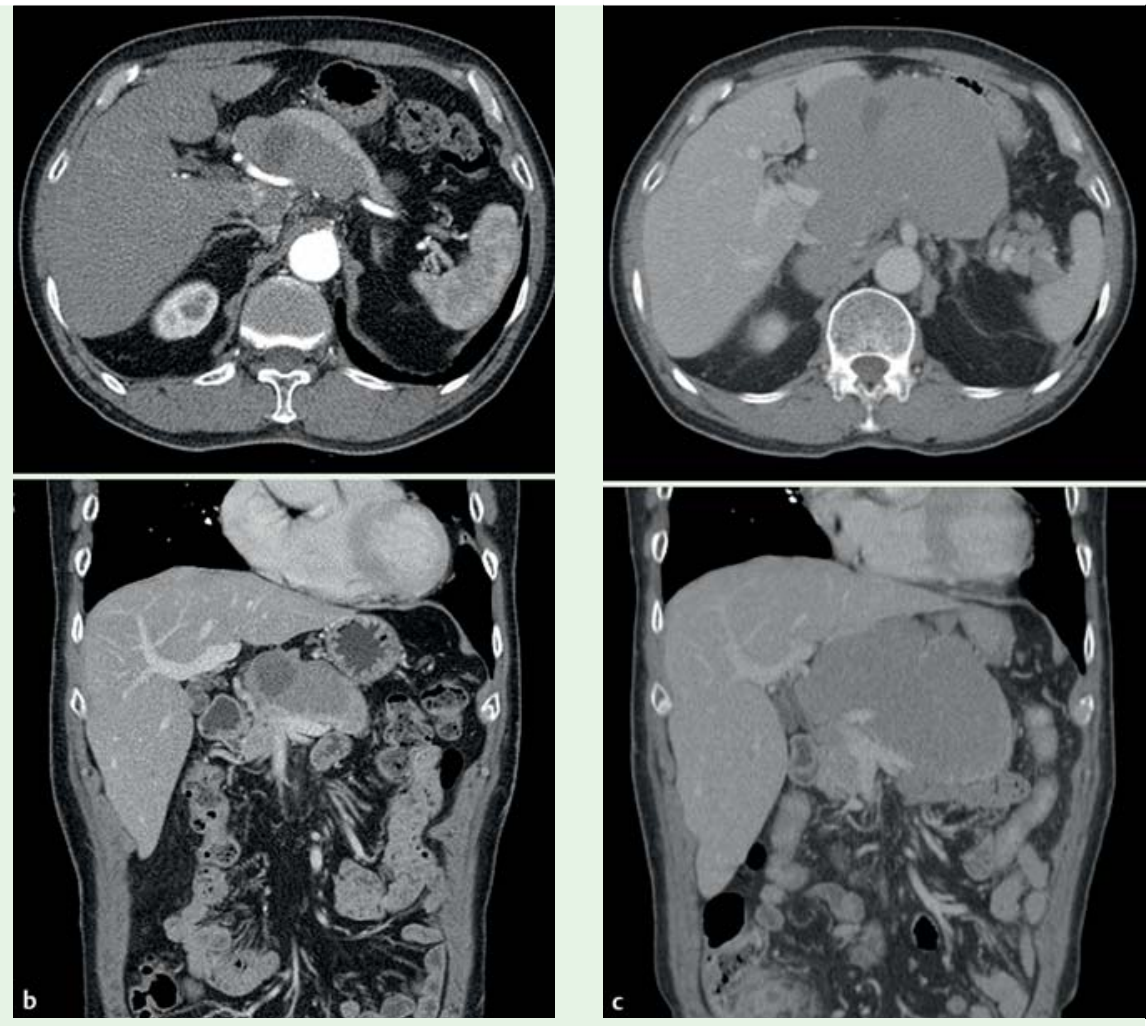

Pancreatic injury, which occurs in approximately $5 \%$ of patients who sustain blunt abdominal trauma, is associated with a morbidity rate of up to $45 \%$ and a mortality rate of up to $30 \%$ [1]. Low grade blunt injuries without main pancreatic ductal damage are initially managed nonoperatively rather than through operative exploration and repair [2]. The differentiation between an organizing hematoma and a mass like a lymphoma is not always straightforward $[3,4]$. Notably, the mass in this case became smaller during the first 6 months. The mantle cell lymphoma, which presented as a slowly growing mass, was stationary in the early stage, and absorption of the hematoma occurred more quickly than growth of the lymphoma [5].

This case highlights the fact that clinicians should continue observation until a hematoma that has arisen at an unusual site completely disappears. If the behavior of a lesion does not correspond to the patient's clinical presentation or exhibits an unusual course, the patient must be evaluated to establish the correct diagnosis.

\section{Endoscopy_UCTN_Code_CCL_1AZ_2AO}

Competing interests: None

Seungho Lee ${ }^{1}$, Joung-Ho Han', Ki Bae Kim', Seon Mee Park', Min Ho Kang², Hye-Suk Han ${ }^{3}$, Song-Yi Choi ${ }^{4}$

${ }^{1}$ Department of Gastroenterology, Chungbuk National University College of Medicine, Cheongju, Republic of Korea 2 Department of Radiology, Chungbuk National University College of Medicine, Cheongju, Republic of Korea ${ }^{3}$ Department of Oncology, Chungbuk National University College of Medicine, Cheongju, Republic of Korea

${ }^{4}$ Departments of Pathology and Oncology, Chungnam National University College of Medicine, Daejeon, Republic of Korea 


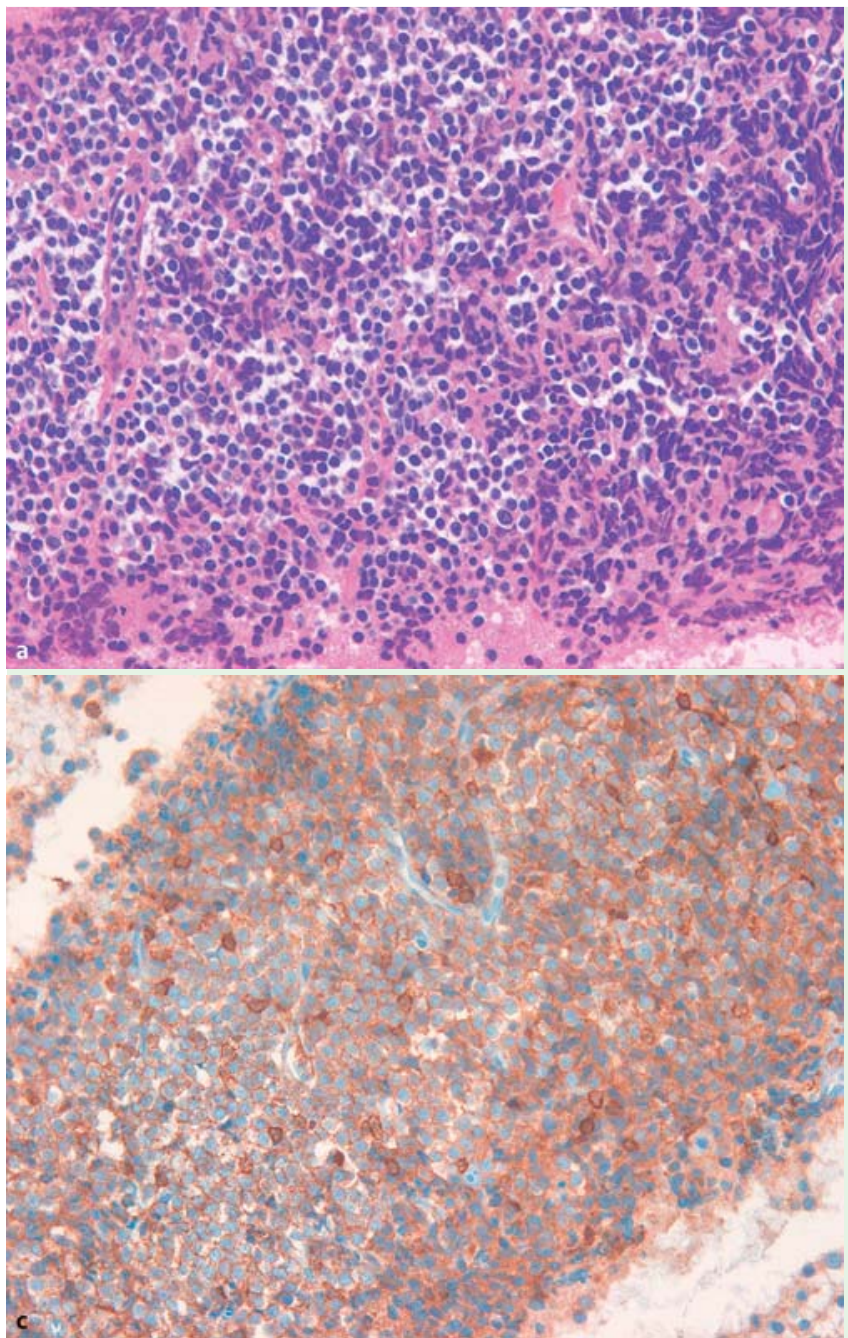

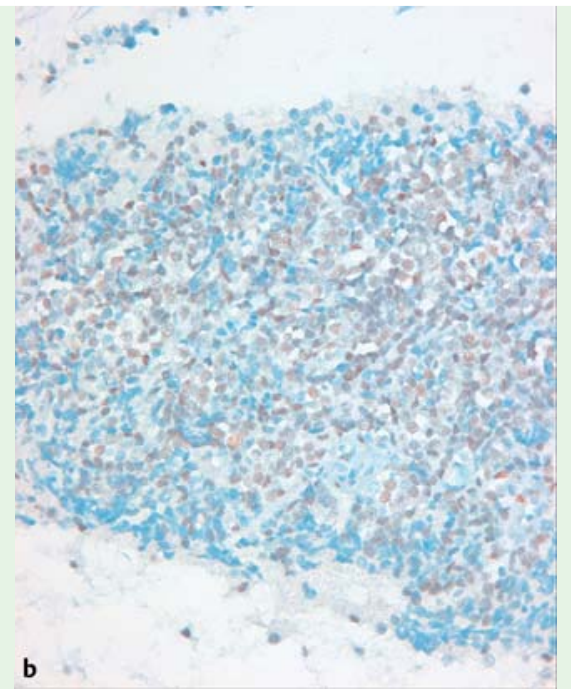

Fig.2 Pathologic examination and immunohistochemical staining of mantle cell lymphoma. a The specimen contains small lymphocytes with irregular nuclei. b, c Immunohistochemical staining reveals positivity for cyclin D1 and CD5. Chungbuk National University College of Medicine 410 SungBong-Ro Heungdeok-Gu Cheongju-Si ChungBuk, 361-711 Republic of Korea Fax: +82-43-273-3252 joungho@cbnu.ac.kr 